\title{
Un nuovo device per la somministrazione di iloprost mediante pompa a siringa portatile: sicurezza, tollerabilità e gradimento
}

\author{
lloprost infusion by a new device as a portable syringe pump: \\ safety, tolerability and agreement
}

\section{Paola Faggioli*, Alba Sciascera, Leopoldo Giani, Antonino Mazzone}

UO Medicina Interna, AO Ospedale Civile di Legnano (MI), Presidio di Legnano

Ricevuto il 4 settembre 2012; accettato il 6 settembre 2012

Disponibile online il 5 ottobre 2012

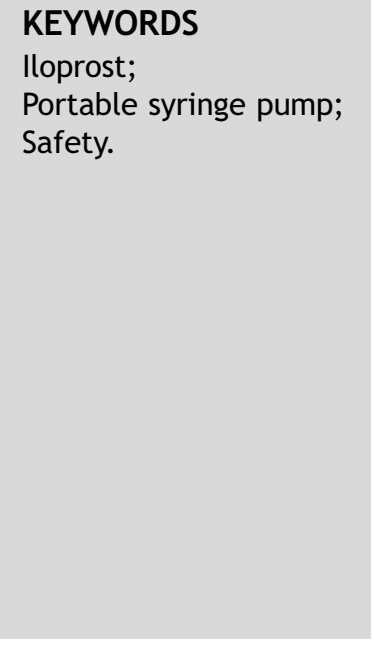

\begin{abstract}
Summary
Background: Iloprost, prostacyclin (PGI2) analogue, effective in treatment of peripheral arterial disease, secondary Raynaud's phenomenon (RP) to connective tissue disease (CTD), vasculitis, pulmonary hypertension, is usually infused through peristaltic pump, or recently through a flow regulator.

Materials and methods: We tested a new portable syringe pump (Pompa Infonde ${ }^{\circledR}$, Italfarmaco S.p.A., Cinisello Balsamo, Milano) on 120 patients affected by RP to CTD and cryoglobulinaemia, in iloprost therapy with a flow regulator.

Results: Iloprost infused through portable syringe pump is better tolerated, better appreciated by the patients and nurses and no difference was observed on therapeutic effects, with a lower incidence of side effects statistically significant. Only 3 patients were unable to tolerate the device ( 2 for changes in pressure and 1 for fear) and shifted to traditional method of iloprost infusion. Conclusions: Iloprost infusion through the portable syringe Pompa Infonde ${ }^{\circledR}$ appears to be safe, better tolerated, more acceptable and equally effective compared to infusion through a flow regulator.

(c) 2012 Elsevier Srl. All rights reserved.
\end{abstract}

\section{Introduzione}

Iloprost, analogo sintetico stabile della prostaciclina naturale (PGI2) [1], è indicato nel trattamento delle principali patologie vascolari di tipo ischemico quali l'arteriopatia obliterante periferica [2,3], la malattia di Buerger [4,5], il fenomeno di Raynaud secondario a sclerosi sistemica [6-9], l'ipertensione polmonare, sia per via inalatoria sia per via

\footnotetext{
* Corrispondenza: Paola Faggioli, Ospedale Civile, via Papa Giovanni Paolo II, 20025 Legnano (MI).

E-mail: medicina2legnano@ao-legnano.it (P. Faggioli).
} 
infusiva [10-13]. Nuovi studi clinici hanno esteso le possibili indicazioni al trattamento di ulcere vasculitiche, sindromi da iperviscosità [14], embolia periferica da cristalli di colesterolo ("blu toes syndrome") [15], trattamento delle ulcere venose $[16,17]$, osteonecrosi asettica $[18,19]$, pioderma gangrenoso [20], post-trapianto [21], prevenzione del vasospamo coronarico [22] e negli stadi precoci delle arteriopatie periferiche e diabetiche [23-25].

A causa della breve emivita di distribuzione tessutale (4 $\mathrm{min}$ ), l'iloprost viene somministrato con un'infusione endovenosa della durata di 6 ore, raggiungendo lo steady state in 20 min, mentre l'emivita di eliminazione è di circa 20-30 min. L'escrezione, per l' $80 \%$ urinaria e per il $20 \%$ epatica, avviene sotto forma di vari metaboliti (di cui il principale è il tetranoriloprost farmacologicamente inattivo), dotati di un'emivita di 2-5 ore a livello plasmatico e 2-18 ore a livello urinario [1].

Secondo lo schema posologico approvato dalle autorità regolatorie, iloprost deve essere infuso a una velocità di 0,5-2 $\mathrm{ng} / \mathrm{kg} / \mathrm{min}$ per almeno 6 ore continuative. Gli schemi terapeutici, sia per durata dei cicli infusionali sia per periodicità, variano a seconda della patologia trattata. Comunemente si consiglia l'uso di una pompa peristaltica per infusione che, garantendo una velocità costante e controllabile, ridurrebbe il rischio di reazioni avverse legate a possibili anomali picchi infusionali.

Iniziali studi condotti sull'infusione di iloprost mediante deflussore con regolatore di flusso in casistiche limitate di pazienti hanno confermato un'efficacia terapeutica e un profilo di sicurezza sovrapponibili a quelli dell'infusione mediante pompa peristaltica [26-28]. In un nostra precedente comunicazione relativa a 18.432 infusioni di iloprost eseguite con regolatore di flusso [29] abbiamo dimostrato che tale metodica appare ugualmente efficace, sicura e ben tollerata, con un'incidenza di effetti collaterali simili a quelli evidenziati con la pompa peristaltica. Tuttavia, anche l'infusione con regolatore di flusso offre limitate possibilità di autonomia al paziente. Scopo del nostro studio, pertanto, è valutare una nuova metodica infusionale di iloprost mediante pompa a siringa portatile al fine di stabilirne profilo di tollerabilità, sicurezza, efficacia e gradimento da parte dei pazienti e del personale infermieristico.

Gli autori dichiarano che lo studio presentato è stato realizzato in accordo con gli standard etici stabiliti nella Dichiarazione di Helsinki, e che il consenso informato è stato ottenuto da tutti i partecipanti prima del loro arruolamento allo studio.

\section{Materiali e metodi}

Da maggio a novembre 2011 sono stati arruolati consecutivamente 120 pazienti afferenti al Day Hospital dell'Unità Operativa di Medicina Interna del Presidio Ospedaliero di Legnano (MI), affetti da fenomeno di Raynaud secondario a malattie del tessuto connettivo (comprendenti sclerosi sistemica, connettivite mista, crioglobulinemia), in terapia con iloprost infuso mediante regolatore di flusso "roller" (Euroset System, Eurospital, Trieste) collegato a un sistema di venipuntura (Neo Venopic 2, G22, Artsana SpA, Grandate, Como), le cui caratteristiche sono illustrate nella tabella 1.
Tabella 1 Caratteristiche dei pazienti.

\begin{tabular}{lc}
\hline Pazienti $(N)$ & 120 \\
\hline Sesso (F/M) & $112 / 8$ \\
Età, anni (mediana) & $54,3 \pm 12,4(52,9)$ \\
Diagnosi & $112(93,3 \%)$ \\
- Sclerosi sistemica, N (\%) & $6(5 \%)$ \\
- Connettivite mista, N (\%) & $2(1,7 \%)$ \\
- Crioglobulinemia, N (\%) & \\
\hline
\end{tabular}

Tutti i pazienti ricevevano iloprost a un dosaggio stabile, e con effetti collaterali costanti, da almeno 2 anni.

I pazienti sono stati sottoposti a infusione di iloprost mediante pompa a siringa portatile, mantenendo lo stesso dosaggio terapeutico. Il device pompa a siringa portatile Pompa Infonde ${ }^{\circledR} \quad$ (Italfarmaco S.p.A., Cinisello Balsamo, Milano) con il raccordo per la pompa (Original Perfusor Line, Braun, Melsungen, Germania) e Delta Connector 3w (Delta Med, Viadana, Mantova) veniva collegato con il sistema di venipuntura Neo Venopic 2 a una vena periferica o al dispositivo venoso centrale (port-a-cath) qualora presente, mediante ago gripper (Gripper Plus, Smiths Medical Italia Srl, Cusago, Milano). La pompa a siringa veniva posta al collo o alla cintura del paziente, che era libero nei movimenti e nella deambulazione.

Si effettuava il monitoraggio della velocità infusionale e degli effetti collaterali.

Ai pazienti veniva somministrato un questionario comprendente quattro item con risposta su scala analogica graduata da 0 a 10 in base a un giudizio totalmente sfavorevole (0) o totalmente favorevole (10) (item: 1. Esperienza con il nuovo device; 2 . Maneggevolezza: 3. Comodità di utilizzo; 4. Effetto farmacologico).

Parallelamente il personale infermieristico compilava, con la stessa modalità visuale analogica numerica, un analogo questionario comprendente quattro item (item: 1. Procedura di preparazione; 2. Modalità di programmazione; 3. Gestione del paziente; 4. Approccio del paziente al device).

Tale studio è stato notificato alla Direzione aziendale, e di conseguenza al Comitato Etico Locale (per le innovazioni tecnologiche).

L'analisi statistica, per quanto limitata, è stata effettuata utilizzando il test del $\chi$ quadrato (Statistical Package for Social Science per Windows).

\section{Risultati}

Per ogni paziente sono stati riportati i comuni effetti collaterali associati all'infusione di iloprost mediante regolatore di flusso e Pompa Infonde ${ }^{\mathrm{B}}$ (tab. 2).

Dai dati emerge una differenza statisticamente significativa per la minore incidenza di ipotensione (0,8\% Pompa Infonde ${ }^{\circledR}$ vs $15 \%$ regolatore di flusso; $p<0,030$ ) e di ipertensione (10\% Pompa Infonde ${ }^{\circledR}$ vs $40 \%$ regolatore di flusso; $\mathrm{p}<0,001)$ clinicamente significative. Per contro, la comparsa di stria iperemica venosa lungo il decorso infusionale appariva superiore per la pompa portatile (94\% Pompa Infonde ${ }^{\circledR}$ vs $60 \%$ regolatore di flusso; $\left.p<0,01\right)$. Gli altri effetti collaterali infusionali apparivano sovrapponibili nelle due metodiche. 
Tabella 2 Effetti collaterali della somministrazione con Pompa Infonde ${ }^{\mathbb{R}}$ vs microgocciolatore.

\begin{tabular}{lcc}
\hline Effetti collaterali & $\begin{array}{c}\text { Pompa Infonde } \\
(\%)\end{array}$ & $\begin{array}{c}\text { Microgocciolatore } \\
(\%)\end{array}$ \\
\hline Flushing & 95 & 92 \\
Stria iperemica venosa & 94 & $60(\mathrm{p}<0,01)^{\mathrm{a}}$ \\
Cefalea & 85 & 90 \\
Vomito/nausea & 60 & 65 \\
Diarrea & 5 & 8 \\
Trisma & 60 & 60 \\
Ipertensione & $10(\mathrm{p}<0,001)^{\mathrm{b}}$ & 40 \\
Ipotensione & $0,8(\mathrm{p}=0,030)^{\mathrm{c}}$ & 15 \\
\hline Note & \\
a Test del $\chi^{2}$ quadrato: odds ratio $=9,36$. & \\
b Test del $\chi^{2}$ quadrato: odds ratio $=0,17$. & \\
c Test del $\chi^{2}$ quadrato: odds ratio $=0,14$.
\end{tabular}

Tre pazienti non hanno tollerato il device: 1 per crisi ipertensiva, 1 per crisi ipotensiva, 1 per paura del device. Tali pazienti hanno continuato la terapia con regolatore di flusso (tab. 3).

I dati relativi al questionario somministrato ai pazienti hanno confermato, per i quattro item, un parere favorevole uguale o superiore al $95 \%$ (tab. 4) e 117 pazienti su 120 hanno chiesto di poter continuare la terapia con il nuovo device.

Il gradimento da parte del personale infermieristico ricavato dalle risposte ai quattro item del questionario (tab. 5) ha confermato valori superiori al $95 \%$ per la modalità di programmazione, la gestione del paziente e l'approccio del paziente, mentre appariva inizialmente meno gradita la procedura di preparazione della pompa, che è stata successivamente semplificata sulla base dei suggerimenti dello stesso personale infermieristico coinvolto.

\section{Discussione e conclusioni}

I nostri dati sembrano confermare sicurezza e tollerabilità della somministrazione di iloprost con pompa a siringa portatile (Pompa Infonde ${ }^{\mathbb{R}}$ ) in rapporto alla somministrazione con regolatore di flusso.

L'utilizzo del nuovo device, inoltre, sembra ridurre l'incidenza di oscillazioni pressorie in corso di infusione.

Nonostante l'efficacia clinica non fosse uno degli endpoint del nostro studio, esso sembra confermare un'uguale efficacia terapeutica per le due metodiche.

Appare significativo il gradimento della nuova metodica da parte sia dei pazienti sia del personale infermieristico. I pazienti ne apprezzano la maneggevolezza, associata alla maggiore autonomia di movimento, la non aumentata incidenza di effetti collaterali infusionali e, forse, la minore percezione di "malattia". Per il personale infermieristico, la possibilità di utilizzare un device che richiede una minore sorveglianza continua potrebbe essere utile ai fini di una migliore gestione delle risorse umane e professionali.

Infine, anche alla luce degli ultimi studi di farmacoeconomia nella gestione dei prostanoidi [30], una metodica infusionale più maneggevole potrebbe, in futuro, offrire maggiori opportunità di impiego di iloprost anche in regime ambulatoriale, consentendo l'accesso a tale terapia a un numero crescente di pazienti.

Tabella 3 Sospensione definitiva del trattamento con Pompa Infonde ${ }^{\circledR}$.

\begin{tabular}{lcl}
\hline Evento & Pazienti $(\mathrm{N})$ & Provvedimento \\
\hline Ipotensione grave $(<100 / 70 \mathrm{mmHg})$ & 1 & Ritorno alla somministrazione con dial-flow \\
Ipertensione grave $(>160 / 100 \mathrm{mmHg})$ & 1 & Ritorno alla somministrazione con dial-flow \\
Paura del device & 1 & Ritorno alla somministrazione con dial-flow \\
\hline
\end{tabular}

Tabella 4 Gradimento della Pompa Infonde ${ }^{\circledR}$ da parte dei pazienti.

\begin{tabular}{lcc}
\hline Domanda & $\begin{array}{c}\text { Punteggio medio } \\
\text { (min-max) }\end{array}$ & $\begin{array}{r}\text { Parere favorevole } \\
\text { dei pazienti (\%) }\end{array}$ \\
\hline 1. Esperienza con il nuovo device & $8,7(7-10)$ & 98 \\
2. Maneggevolezza (nuovo device vs infusione con dial-flow) & $9(8-10)$ & 99 \\
3. Comodità di utilizzo & $8,9(7-10)$ & 95 \\
4. Effetto farmacologico & $9,1(8-10)$ & 97 \\
\hline
\end{tabular}

Tabella 5 Gradimento della Pompa Infonde ${ }^{\circledR}$ da parte del personale infermieristico.

\begin{tabular}{lcc}
\hline Domanda & $\begin{array}{c}\text { Punteggio medio } \\
(\text { min-max })\end{array}$ & $\begin{array}{c}\text { Parere favorevole del personale } \\
\text { infermieristico (\%) }\end{array}$ \\
\hline 1. Procedura di preparazione & $7,5(6-10)$ & 90 \\
2. Modalità di programmazione & $8,1(6-9)$ & 98 \\
3. Gestione del paziente & $8,6(7-10)$ & 95 \\
4. Approccio del paziente al device & $9,8(9-10)$ & 98 \\
\hline
\end{tabular}




\section{Conflitto di interessi}

Gli autori dichiarano di non aver alcun conflitto di interessi.

\section{Ringraziamenti}

Gli autori ringraziano: G. Faietti, S. Solinas, A. Gattolin, G. dalle Carbonare, A. Sindaco (infermieri professionali del Day Hospital di Medicina Interna dell'Azienda Ospedaliera Ospedale Civile di Legnano.

\section{Bibliografia}

[1] Wada M, Yokoyama C, Hatae T, Shimonishi M, Nakamura M, Imai $Y$, et al. Purification and characterization of recombinant human prostacyclin synthase. J Biochem 2004;135(4):455-63.

[2] Hirsch AT, Haskal ZJ, Hertzer NR, Bakal CW, Creager MA, Halperin JL, et al., American Association for Vascular Surgery; Society for Vascular Surgery; Society for Cardiovascular Angiography and Interventions; Society for Vascular Medicine and Biology; Society of Interventional Radiology; ACC/AHA Task Force on Practice Guidelines Writing Committee to Develop Guidelines for the Management of Patients with Peripheral Arterial Disease; American Association of Cardiovascular and Pulmonary Rehabilitation; National Heart, Lung, and Blood Institute; Society for Vascular Nursing; TransAtlantic InterSociety Consensus; Vascular Disease Foundation. ACC/AHA 2005 practice guidelines for the management of patients with peripheral arterial disease (lower extremity, renal, mesenteric, and abdominal aortic). Circulation 2006;113(11): e463-654.

[3] de Donato G, Gussoni G, Cao P, Setacci C, Pratesi C, Mazzone A, et al., ILAILL Study Group. Acute limb ischemia in elderly patients: can iloprost be useful as an adjuvant to surgery? Results from the ILAILL study. Eur J Vasc Endovasc Surg 2007;34(2):194-8.

[4] Norgren L, Hiatt WR, Dormandy JA, Nehler MR, Harris KA, Fowkes FG, et al., TASC II Working Group. Inter-society consensus for the management of peripheral arterial disease. Int Angiol 2007;26(2):81-157.

[5] Afsharfard A, Mozaffar M, Malekpour F, Beigiboroojeni A, Rezaee M. The wound healing effects of iloprost in patients with Buerger's disease: claudication and prevention of major amputations. Iran Red Crescent Med J 2011;13(6):420-3.

[6] Scorza R, Caronni M, Mascagni B, Berruti V, Bazzi S, Micallef E, et al. Effects of long-term cyclic iloprost therapy in systemic sclerosis with Raynaud's phenomenon. A randomized, controlled study. Clin Exp Rheumatol 2001;19(5):503-8.

[7] D'Amelio P, Cristofaro MA, D'Amico L, Veneziano L, Roato I, Sassi $\mathrm{F}$, et al. lloprost modulates the immune response in systemic sclerosis. BMC Immunol 2010;11:62.

[8] Stratton R, Shiwen X, Martini G, Holmes A, Leask A, Haberberger $\mathrm{T}$, et al. Iloprost suppresses connective tissue growth factor production in fibroblasts and in the skin of scleroderma patients. J Clin Invest 2001;108(2):241-50.

[9] Casigliani Rabl S, Della Rossa A, Pepe P, D'Ascanio A, Mosca M, Di Vita A, et al. Long-term cyclic intravenous iloprost in systemic sclerosis: clinical experience from a single center. Reumatismo 2012;64(3):158-65.

[10] Olschewski H, Hoeper MM, Behr J, Ewert R, Meyer A, Borst MM, et al. Long-term therapy with inhaled iloprost in patients with pulmonary hypertension. Respir Med 2010;104(5):731-40.

[11] Caramaschi P, Dalla Gassa A, Prati D, Barausse G, Tinazzi I, Ravagnani V, et al. Severe vascular complications in patients affected by systemic sclerosis cyclically treated with iloprost. Rheumatol Int 2012;32(7):1933-8.

[12] Caravita S, Wu SC, Secchi MB, Dadone V, Bencini C, Pierini S. Long-term effects of intermittent iloprost infusion on pulmonary arterial pressure in connective tissue disease. Eur J Intern Med 2011;22(5):518-21.

[13] Sheffield Teaching Hospitals NHS Foundation Trust. Iloprost in Pulmonary Arterial Hypertension 2011.

[14] Zulian F, Costantini C, Montesco MC, Schiavon F, Zacchello F. Successful treatment of gangrene in systemic necrotizing vasculitis with iloprost. Br J Rheumatol 1998;37(2):228-30.

[15] Meyrier A. Cholesterol crystal embolism: diagnosis and treatment. Kidney Int 2006;69(8):1308-12.

[16] Ferrara F, Meli F, Raimondi F, Amato C, Bonura F, Mulè G, et al. The treatment of venous leg ulcers: a new therapeutic use of iloprost. Ann Surg 2007;246(5):860-5.

[17] Canciglia A, Mandolfino T, D'Alfonso M. The efficacy of iloprost for the treatment of chronic venous ulcers of the lower limbs. G Chir 2011;32(10):434-7.

[18] Jäger $M$, Zilkens $C$, Bittersohl $B$, Matheney T, Kozina $G$, Blondin D, et al. Efficiency of iloprost treatment for osseous malperfusion. Int Orthop 2011;35(5):761-5.

[19] Meini S, Panigada G. Proposal of early retreatment with iloprost in partially responsive patients with bone marrow edema syndrome: a case report. It Journ Med; in press. Available online 12 June 2012.

[20] Mazzone A, Cusa C, Mazzucchelli I, Rosso R, Vezzoli M. Pyoderma gangrenosum complicating ulcerative colitis: An alternative treatment with iloprost. Current Therapeutic Research 1999;60:357-62.

[21] Bärthel E, Rauchfuss F, Hoyer H, Habrecht O, Jandt K, Götz M, et al. Impact of stable PGI2 analog iloprost on early graft viability after liver transplantation: a pilot study. Clin Transplant 2012;26(1):E38-47.

[22] Ege T, Gur O, Karadag CH, Duran E. Evaluation of iloprost to prevent vasospasm in coronary artery bypass grafts. J Int Med Res 2010;38(5):1759-63.

[23] Duthois S, Cailleux N, Benosman B, Lévesque H. Tolerance of iloprost and results of treatment of chronic severe lower limb ischaemia in diabetic patients. A retrospective study of 64 consecutive cases. Diabetes Metab 2003;29(1):36-43.

[24] Meini S, De Franco V, Auteri A, Setacci C, Di Renzo M, Pieragalli D. Short-term and long-term effects of one-week treatment with intravenous iloprost in critical limb ischemia patients (Leriche-Fontaine stage III and IV). Int Angiol 2005;24(1):64-9.

[25] Piaggesi A, Vallini V, lacopi E, Tedeschi A, Scatena A, Goretti C, et al. lloprost in the management of peripheral arterial disease in patients with diabetes mellitus. Minerva Cardioangiol 2011;59(1):101-8.

[26] Veroux P, Veroux M, Macarone M, Bonanno MG, Tumminelli MG. Efficacy of a novel method of intravenous infusion of the prostaglandin analogue iloprost for the treatment of lower-limb critical ischemia: an open-label, nonrandomized study in two cohorts. Current Therapeutic Research 2004;65(3):255-65.

[27] Mazzone A, Vezzoli M, Ottini E, Montagna M, Mazzucchelli I, Dal Canton A. A new method of iloprost administration without a peristaltic pump. Current Therapeutic Research 2000;61:452-9.

[28] Arreghini M, Prudente P, Maglione W, Arnoldi C, Tosi S, Marchesoni A. Tolerability, safety and efficacy of iloprost infusion without peristaltic pump in systemic sclerosis. Reumatismo 2001;53(2):140-4.

[29] Faggioli P, Giani L, Mazzone A. Sicurezza e tollerabilità della somministrazione di iloprost senza pompa peristaltica. It J Med 2010;4(3):179-84.

[30] Roman A, Barberà JA, Escribano P, Sala ML, Febrer L, Oyagüez I, et al. Cost effectiveness of prostacyclins in pulmonary arterial hypertension. Appl Health Econ Health Policy 2012;10(3):175-88. 